\section{Thermal Maps of GaAs P-HEMT: A Novel System Based on the Photocurrent Spectral Analysis}

\author{
Andrea Reale, Aldo Di Carlo, Marco Peroni, \\ Claudio Lanzieri, and S. Lavagna
}

\begin{abstract}
In this brief, we present a novel noninvasive method for spatially resolved thermal measurement of HEMT devices based on microphotoconductance analysis. This approach is used to obtain the temperature distribution in the active regions of a GaAs P-HEMT. Through 1-D and 2-D thermal maps, we are able to measure the temperature inside each single channel, and owing to the improved spatial resolution of the developed technique, it is possible to observe the hottest region of the device which is placed at the drain side of the gate. Moreover, the resolution of the temperature measurements allows to define a local thermal resistance which is not uniform over the device due to the mutual heating between the channels.
\end{abstract}

Index Terms-Device heating, P-HEMT, spatially resolved photoconductance, thermal resistance.

\section{INTRODUCTION}

In transistor devices designed for high-power applications, the temperature of the active region is a very important parameter for the output power performance, since output current can be limited by channel heating effects [1]. Moreover, heating aspects are very important in terms of failure rate which, in devices like HEMTs, is mainly determined by the operating temperature of the channel [2]. Thermal management of high-power devices represents a critical aspect in device design. A detailed knowledge of self-heating effects and an accurate study of the power dissipation are thus needed, as well as the optimization of the geometrical layout and of the substrate mounting technology.

Several measurement techniques of device temperature are commonly used: electrical dc method [3], IR techniques [4], liquid crystal techniques [5], Raman spectroscopy [6], cathodoluminescence [7], and photoluminescence spectroscopy [8]. Many of these methods do not allow the exact evaluation of the channel temperature, since some of these techniques (such as [4] and [5]) are able to measure only the surface temperature of the device, while some other techniques (such as [7] and [8]) may be affected by carrier diffusion phenomena that tend to smear out the knowledge of temperature distribution. Some others (such as [6]) are able to achieve an in-plane $x-y$ accuracy slightly higher than our technique; however, this technique is based on the measurement of the optical phonon frequency of the GaN layer, and thus, it provides an averaged temperature information across the transversal growth direction due to the optical response of all the bulk $\mathrm{GaN}$ materials to the laser radiation. Recently, we have presented an efficient simple noninvasive method [9]-[11] of evaluating the actual channel temperature of FET devices based on the measurement of the photocurrent spectrum. The advantage of this method consists of the capability of measuring directly the temperature of the HEMT

Manuscript received August 7, 2006; revised December 27, 2006. The review of this brief was arranged by Editor M. Anwar.

A. Reale and A. Di Carlo are with the Electronic Engineering Department, University of Rome Tor Vergata, 00133 Rome, Italy (e-mail: reale@ing.uniroma2.it; dicarlo@ing.uniroma2.it).

M. Peroni, C. Lanzieri, and S. Lavagna are with the Department of GaAs, SELEX Sistemi Integrati, 00133 Rome, Italy (e-mail: mperoni@selex-si.com; clanzieri@selex-si.com; slavagna@selex-si.com).

Color versions of one or more of the figures in this brief are available online at http://ieeexplore.ieee.org.

Digital Object Identifier 10.1109/TED.2007.891868

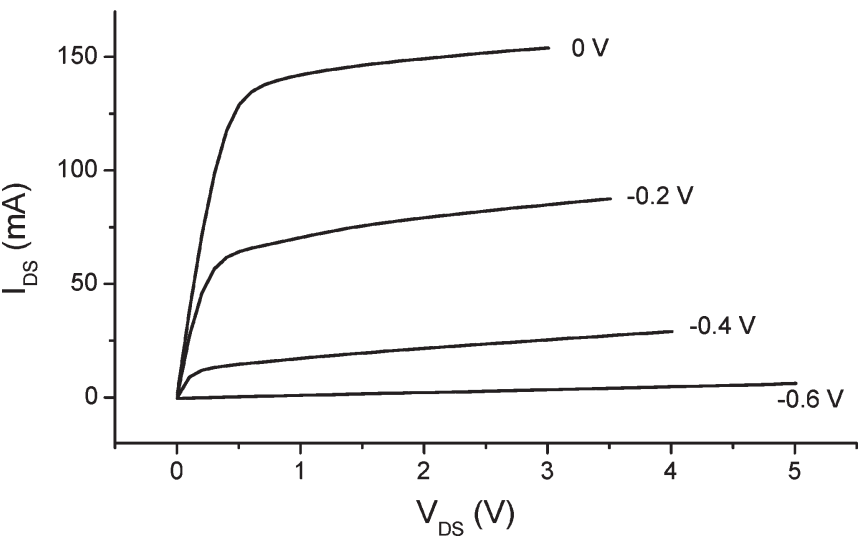

Fig. 1. $I-V$ characteristics of the P-HEMT device. Pinch-off occurs at $V_{\mathrm{GS}}=-0.6 \mathrm{~V}$.

channel, even if this is well below the surface due to the fact that the collection efficiency of the channel is much higher. Thus, the photocurrent spectra are mainly related to the channel conditions. Moreover, carrier diffusion after photoexcitation does not affect the maximum achievable resolution.

In our previous works, we reported the photocurrent thermal measurements of HEMTs by uniform direct illumination [9]-[11]. In this brief, we extend the method to a spatially resolved photoconductance setup, where we focus the light through a single-mode fiber-optic system. The use of a fiber as exciting source allows us to estimate the temperature on an area of the order of fiber mode field diameter (less than $5 \mu \mathrm{m}$ ). The actual achievable resolution depends on the mode field diameter of the fiber we use (typically around $3 \mu \mathrm{m}$ ) and on the distance between the facet of the fiber and the surface of the device.

\section{GaAs P-HEMT DEVICES}

GaAs power P-HEMT devices have been fabricated by SELEX Sistemi Integrati S.p.A. on GaAs/AlGaAs/InGaAs heterostructures epitaxially grown on semi-insulating GaAs substrates. The manufacturing process involves ohmic contact formation obtained by metal deposition of $\mathrm{Au} / \mathrm{Ge} / \mathrm{Ni}$ and subsequent rapid thermal annealing, active device isolation by ion implantation, gate recess wet etching, Schottky barrier metallization ( $\mathrm{Ti} / \mathrm{Al}$ ) for gate contact formation, and surface passivation by plasma enhanced chemical vapour deposition silicon nitride. Finally, the substrate is thinned down to $100 \mu \mathrm{m}$, and via holes are realized through the backside to connect all the source pads. Measured devices have $10 \times 100 \mu \mathrm{m}$ total gate periphery, drain-to-source distance of $5 \mu \mathrm{m}$, gate pitch of $30 \mu \mathrm{m}$, and gate length of $0.6 \mu \mathrm{m}$. The devices have been then placed in a 70MIL package and bonded by using silver epoxy. Current-voltage $(I-V)$ characteristics are shown in Fig. 1. The main RF parameters at $5 \mathrm{GHz}$ are output power of $29.7 \pm 0.1 \mathrm{dBm}$, linear gain of $14.6 \pm$ $0.4 \mathrm{~dB}$, and power-added efficiency of $56 \pm 1 \%$ for $V_{\mathrm{DS}}=9 \mathrm{~V}$, $I_{\mathrm{DS}}=180 \mathrm{~mA}$.

\section{Spatially Resolved Photocurrent SetuP}

In order to obtain a spatially resolved photocurrent spectrum, we realized the setup shown in Fig. 2. The light emitted from a halogen lamp is dispersed by a monochromator, choppered and focused into a single-mode (cutoff wavelength of $370 \mathrm{~nm}$, mode field diameter of $3.2 \mu \mathrm{m}$ ) fiber optic. The end of the fiber is mounted on a 3-D motorized 


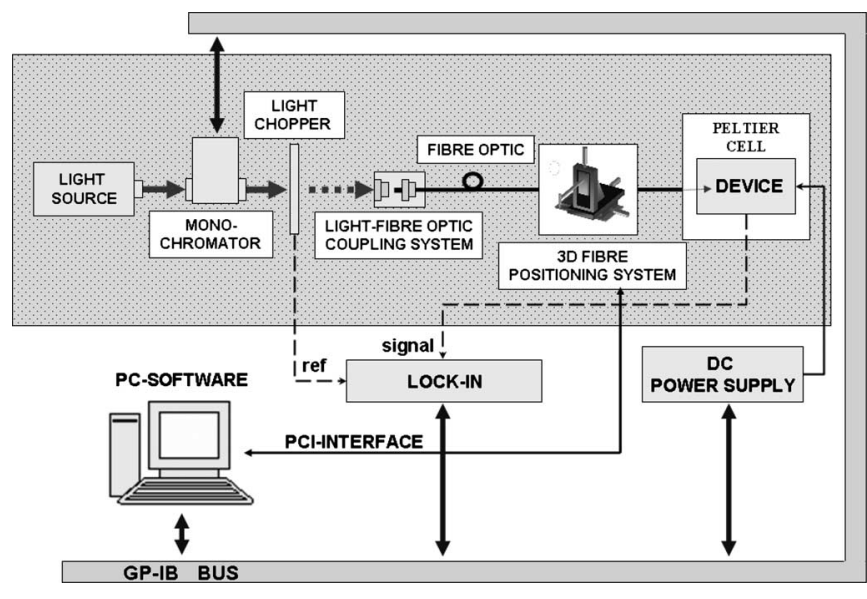

Fig. 2. Spatially resolved photocurrent setup.

positioning system in order to focus the spot which comes from the fiber on the device-channel region.

The minimum increment along the $x$ - and $y$-axes of each motor is $50 \mathrm{~nm}$. The position of the light spot is monitored by optical microscopy, which allows also the control of the fiber-channel distance. This fundamental parameter affects the effective spatial resolution, which can be greater or equal to the mode field diameter of the fiber.

The spatially resolved analysis requires a coherent detection via lock-in techniques of the photocurrent induced on the device. A voltage supply is used to bias the device during the power-dissipation measurements. A Peltier plate is used to fix the temperature $\left(T_{\mathrm{P}}\right)$ of the device carrier. The whole setup is remotely controlled by computer through the dedicated Labview virtual instrument.

\section{Thermal Map of GaAs P-HEMT}

Thermal device performance can be evaluated in terms of the static thermal resistance obtained from the channel temperature as a function of the dissipated power.

In order to calculate the channel temperature, a thermal calibration is necessary. The device is biased with low dissipated-power condition ( $\left.V_{\mathrm{GS}}=0 \mathrm{~V}, V_{\mathrm{DS}}=0.1 \mathrm{~V}, I_{\mathrm{DS}}=40 \mathrm{~mA}\right)$. Then, the temperature of the system is varied via Peltier cell. An increase of $T_{\mathrm{P}}$ causes the red shift of photocurrent spectra due to the temperature dependence of the band gap.

We define $\lambda_{\mathrm{TH}}$ as the wavelength that corresponds to a particular interpolated value of normalized photocurrent spectrum which represents the edge of absorption spectrum (Fig. 3). In the following, we will consider as threshold wavelength the one which corresponds to the $70 \%$ of the maximum photocurrent. Other criteria can be used to define the adsorption edge, but we checked, and this does not change the final results (thermal maps). Calibration provides the relation between the temperature and the wavelength. In the temperature range considered in this brief $\left(20{ }^{\circ} \mathrm{C}-100{ }^{\circ} \mathrm{C}\right)$, we found the following calibration expression: $T_{\mathrm{CH}}[K]=\left(\lambda_{\mathrm{TH}}-363.86[\mathrm{~nm}]\right) / 0.286[\mathrm{~nm} / \mathrm{K}]$, where $T_{\mathrm{CH}}$ is the temperature of the channel of the device. The accuracy of the temperature measurement depends mainly on the uncertainty in the intensity of the photocurrent spectrum (the uncertainty in the spectral position is less than $1 \AA$ ) and on the slope of the spectrum at the 0.7 threshold. Each value of the photocurrent at any given wavelength is averaged through the use of the lock-in detection, with a root-meansquare error $\Delta I$ less than $2 \%$ of the maximum intensity detected in the whole photocurrent spectrum. The typical slope is $\alpha=0.075 \mathrm{~nm}^{-1}$. The resulting uncertainty in the threshold wavelength is $\Delta \lambda_{\mathrm{TH}}=$

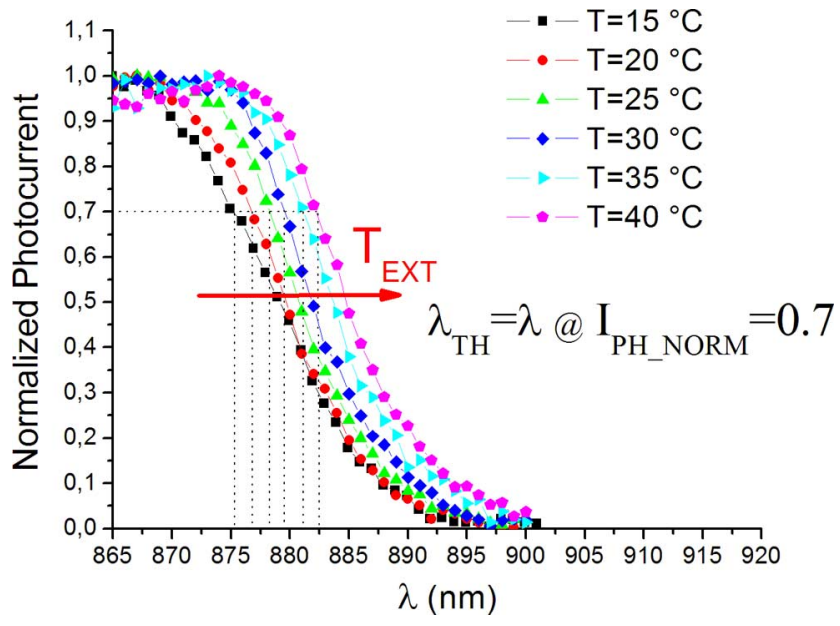

Fig. 3. Shift of the absorption edge of the photocurrent spectra of a GaAs P-HEMT occurring when the external temperature changes $\left(V_{\mathrm{GS}}=0 \mathrm{~V}\right.$, $\left.V_{\mathrm{DS}}=0.1 \mathrm{~V}, I_{\mathrm{DS}}=40 \mathrm{~mA}\right)$.

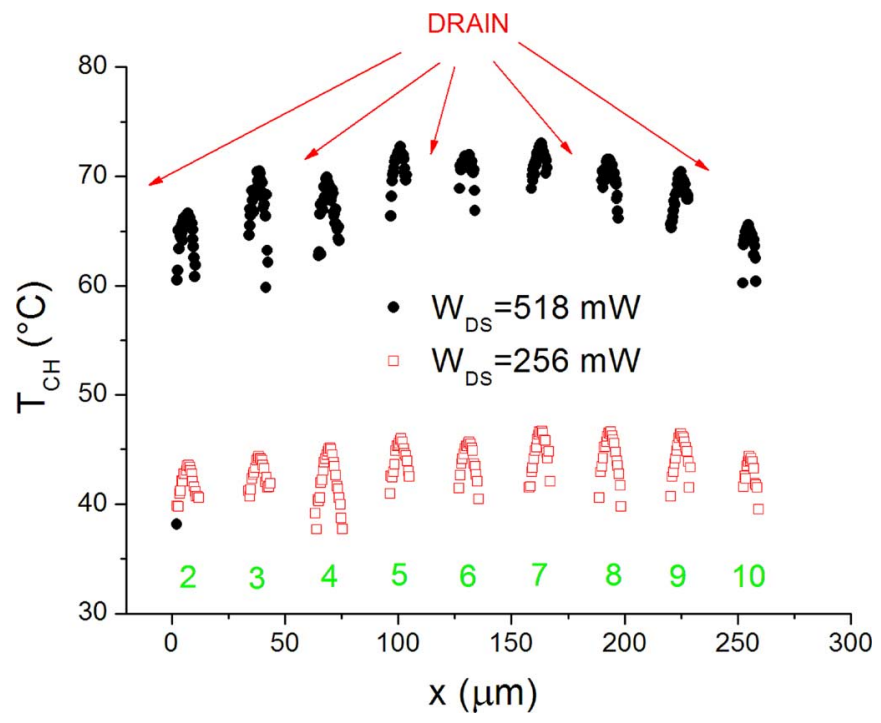

Fig. 4. Linear profile of the temperature of nine channels at different $\mathrm{dc}$ power-dissipation conditions: 1) $W_{\mathrm{DS}}=256 \mathrm{~mW}$ with $V_{\mathrm{GS}}=0 \mathrm{~V}$ and $V_{\mathrm{DS}}=1.8 \mathrm{~V}$, and 2) $W_{\mathrm{DS}}=518 \mathrm{~mW}$ with $V_{\mathrm{GS}}=0 \mathrm{~V}$ and $V_{\mathrm{DS}}=3.5 \mathrm{~V}$.

$1 / \alpha \cdot \Delta I=0.266 \mathrm{~nm}$. This corresponds to a temperature uncertainty of $0.9{ }^{\circ} \mathrm{C}$, according to the aforementioned calibration law.

It is possible to make a thermal calibration on a single point of the device, just focusing the spot at the desired position along one of the channels. We checked, however, that the calibration law is independent on the spot position, since it is only related to the intrinsic properties of the semiconductor material.

For each coordinate $(x, y)$, a photocurrent spectrum is acquired. In this way, we get a $\lambda_{\mathrm{TH}}$ for every point, and through the thermal calibration law, we determine the actual channel temperature $T_{\mathrm{CH}}(x, y)$. A 1-D scan of the device is performed moving the light spot along the channel direction. Fig. 4 shows the temperature distribution along the channels of the device at two different dc dissipated powers. Fig. 5 represents the details of the 1-D temperature distribution of two channels. These measurements have been performed with $T_{\mathrm{P}}=25^{\circ} \mathrm{C}$.

For each channel, the photocurrent spectra are collected over a region which results form the spatial convolution between the mode field diameter of the fiber and the real length of the channel. For this 


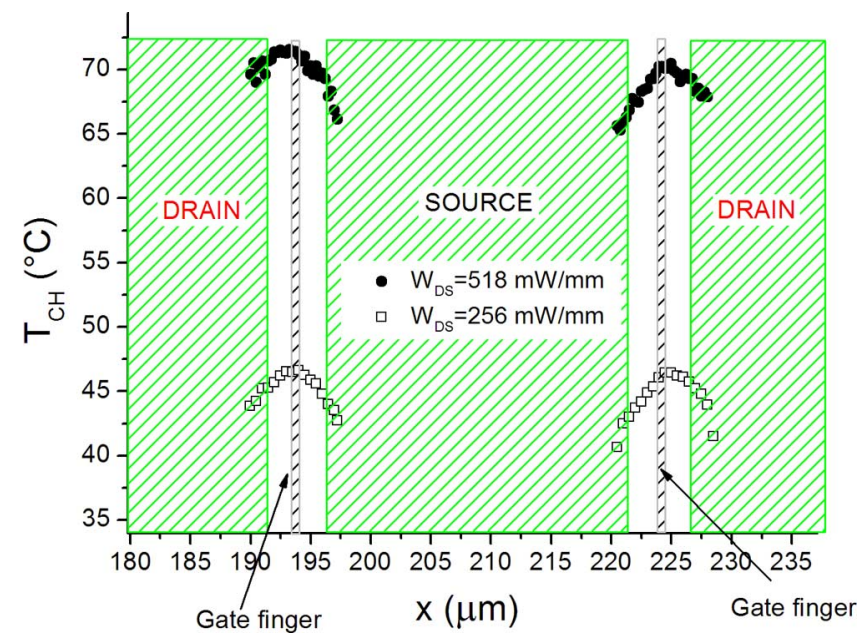

Fig. 5. Particular details of channel temperature in two adjacent channels: It can be observed that the zones between the gate and the drain have a major temperature.
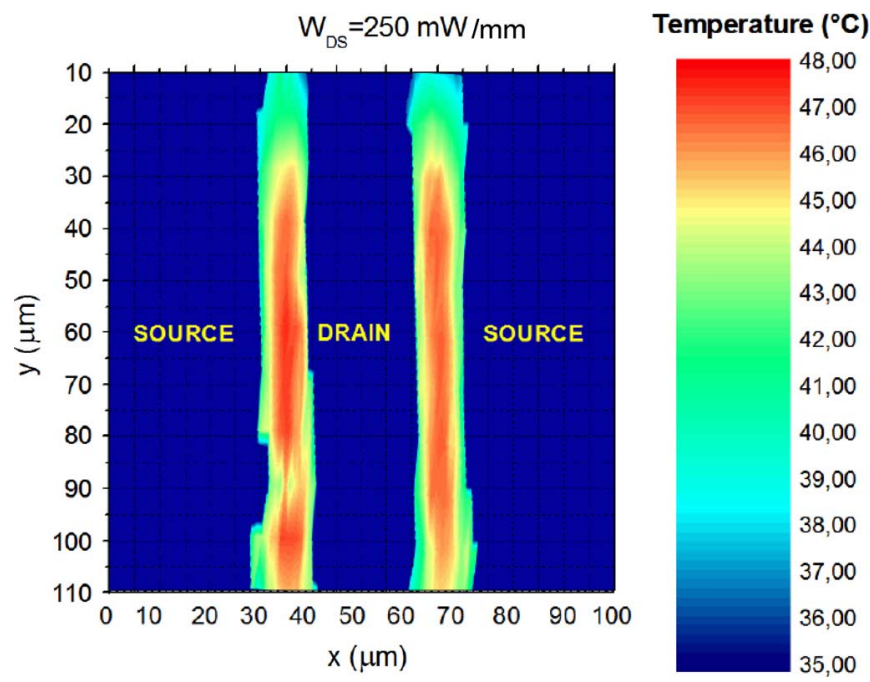

Fig. 6. Two-dimensional temperature map of two GaAs P-HEMT channels (third and fourth of Fig. 5) at $W_{\mathrm{DS}}=256 \mathrm{~mW}$ with $V_{\mathrm{GS}}=0 \mathrm{~V}$ and $V_{\mathrm{DS}}=1.8 \mathrm{~V}$.

reason, the measurements shown in Figs. 4 and 5 slightly extend over the drain and source metallizations.

This technique allows us to profile the temperature variations between the source and the drain. Fig. 5 clearly shows an asymmetry in the channel temperature, since the channel is hotter toward the gate-drain region. Such behavior is more evident at the higher dissipated power. This asymmetry within the device channel can be explained with the positioning of the hottest spot in correspondence with the higher electric field zone, located at the drain side of the gate junction, as well known from several reported simulations [12] and measured [13] data.

Two-dimensional temperature maps can be obtained in a similar way by scanning the surface of the HEMT in both $x$ - and $y$-axes. At each scanning point, a full photocurrent spectrum is recorded, and local temperature is extracted via the calibration equation. Fig. 6 shows the interpolated data measured with constant steps along $x$ - and $y$ directions $(\Delta x=0.5 \mu \mathrm{m}$ and $\Delta y=10 \mu \mathrm{m})$. The real time control of the software provides to exclude measurements at regions covered by metallization in order to reduce the measurement time.

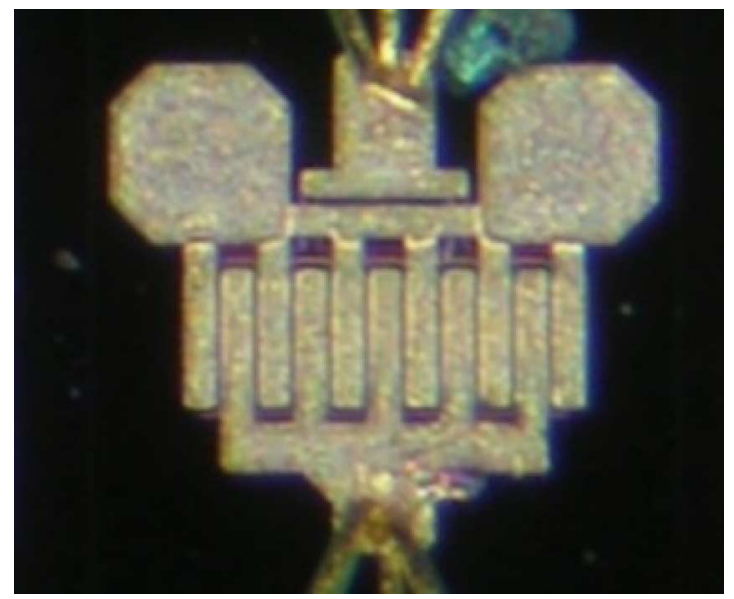

Fig. 7. One-millimeter GaAs P-HEMT power device.

It can be seen that the temperature is greatest in the center of the channel and toward the drain region. Moreover, the heat distribution along the channel is not perfectly symmetrical. The lower region is hotter than the upper region which is in the proximity of via holes. The layout of the device is shown in Fig. 7.

\section{THERMAL RESISTANCE}

In the previous section, we have shown the thermal maps for a given dc bias. However, we can fix the measurement point and record the temperature variation as a function of the dc bias (i.e., the power dissipated in the device). These measurements have been performed for two reference channels (channels 5 and 10) with the light spot focused at the center of the channel. We found, in the bias range considered in this brief, a linear relation between the channel temperature and the dissipated power.

The usual concept of thermal resistance describes macroscopically the process of heating transfer, while the physical parameter that determines how, on a microscopic scale, the temperature distribution is related to the power dissipated in the device is the thermal conducibility. If one is interested to summarize the information of a local temperature distribution among the different channels of the device for a given dissipated power $W_{\mathrm{DS}}$, one can define a local thermal resistance $R_{\mathrm{TH}}$ for any given active region of the device (not covered by metallization) according to the following definition:

$$
R_{\mathrm{TH}}(x, y)=\frac{\Delta T_{\mathrm{CH}}(x, y)}{\Delta W_{\mathrm{DS}}}
$$

where $(x, y)$ is any arbitrary position inside the channel, $W_{\mathrm{DS}}=V_{\mathrm{DS}}$. $I_{\mathrm{DS}}$ is the dc power dissipated within each channel, and $\Delta T_{\mathrm{CH}}=$ $T_{\mathrm{CH}}-T_{\mathrm{P}}$. We observed that the thermal resistance changes from channel to channel. In this case, a central channel (channel 5) has a thermal resistance $R_{\mathrm{TH}}=92{ }^{\circ} \mathrm{C} / \mathrm{W}$, while a peripherical one (channel 10) presents $R_{\mathrm{TH}}=84{ }^{\circ} \mathrm{C} / \mathrm{W}$, as shown in Fig. 8. In this way, we explain the temperature differences shown in Fig. 4, where the central channels are hotter than the peripherical ones. In this way, temperature disuniformities between the different channels of the device can be more easily ascribed to the effect of the geometrical layout and of the packaging of the device.

\section{CONCLUSION}

We have presented a new technique for the spatially resolved measurement of the temperature in HEMT device through the microphotocurrent technique where the light is focused on the channel through 


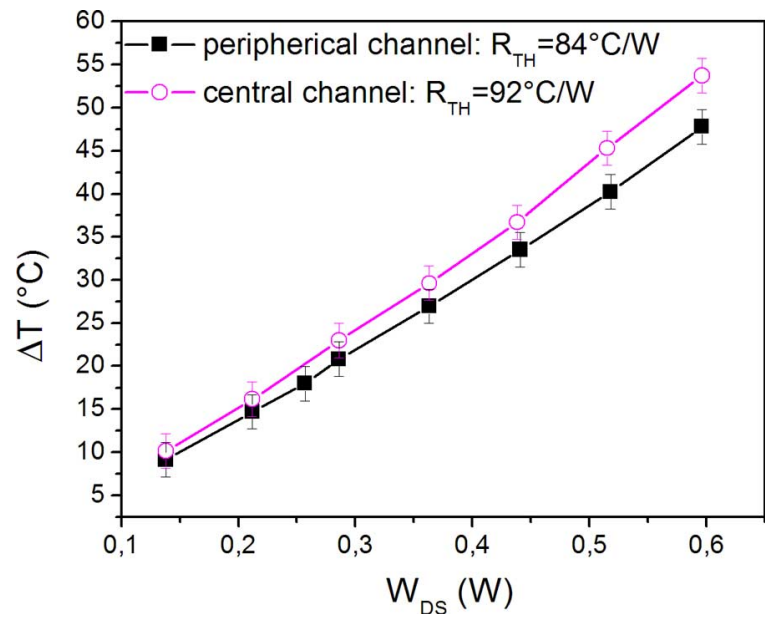

Fig. 8. Heating of channel 5 compared with channel 10. The central channel has an $R_{\mathrm{TH}}$ greater than the peripherical one.

a UV-single-mode fiber optic. This technique has been applied to measure the 1-D and 2-D temperature distributions of GaAs P-HEMTs. We have been able to determine the temperature in each single point of the device channels and even to determine the asymmetry of temperature distribution between the source and the drain.

We have measured the thermal resistance in each single point of the device. The thermal resistance is a local property of the channel and depends on the device layout and package.

\section{ACKNOWLEDGMENT}

The authors would like to thank P. Mencherini and D. Angelilli for the assistance in the photocurrent measurements.

\section{REFERENCES}

[1] J. V. Dilorenzo and W. R. Wisseman, "GaAs power MESFET's: Design, fabrication, and performance," IEEE Trans. Microw. Theory Tech., vol. MTT-27, no. 5, pp. 367-378, May 1979.

[2] W.-C. Liu, K.-H. Yu, R.-C. Liu, K.-W. Lin, K.-P. Lin, C.-H. Yen, C.-C. Cheng, and K.-B. Thei, "Investigation of temperature-dependent characteristics of an $\mathrm{n}+-\mathrm{InGaAs} / \mathrm{n}-\mathrm{GaAs}$ composite doped channel HFET," IEEE Trans. Electron Devices, vol. 48, no. 12, pp. 2677-2683, Dec. 2001.

[3] D. B. Estreich, "A DC technique for determining GaAs MESFET thermal resistance," IEEE Trans. Compon., Hybrids, Manuf. Technol., vol. 12, no. 4, pp. 675-679, Dec. 1989. [see also IEEE Trans. on Components, Packaging, and Manufacturing Technology, Part A, B, C].

[4] H. C. Huang, F. N. Sechi, and L. S. Napoli, "Thermal resistance of GaAs power FETs," in Proc. 6th Biennial Cornell Elect. Eng. Conf., 1977, pp. 297-303.

[5] E. M. Fleuren, "A very sensitive, simple analysis technique using nemaic liquid crystals," in Proc. 25th Int. Reliab. Phys. Symp., San Diego, CA, 1987, vol. 25, p. 369.

[6] M. Kuball, J. M. Hayes, M. J. Uren, I. Martin, J. C. H. Birbeck, R. S. Balmer, and B. T. Hughes, "Measurement of temperature in active high-power AlGaN/GaN HFETS using Raman spectroscopy," IEEE Electron Device Lett., vol. 23, no. 1, pp. 7-9, Jan. 2002.

[7] H. M. Kim, J. S. Yoon, S. H. Lee, J. E. Oh, W. S. Lee, K. I. Chun, K. W. Chung, H. W. Chung, and T. W. Kang, "New technique for the thermal resistance measurement of power field effect transistors using cathodoluminescence," Jpn. J. Appl. Phys., vol. 39, no. 11A, pp. L1087L1089, 2000.

[8] J. P. Landesman, B. Depret, A. Fily, J. Nagle, and P. Braun, "Local channel temperature measurements on pseudomorphic high electron mobility transistors by photoluminescence spectroscopy," Appl. Phys. Lett., vol. 72, no. 11, pp. 1338-1340, Mar. 1998.

[9] D. Savian, A. Di Carlo, R. Lugli, M. Peroni, C. Cetronio, C. Lanzieri, G. Meneghesso, and E. Zanoni, "Channel temperature measurement of
PHEMT by means of optical probes," Electron. Lett., vol. 39, no. 2, pp. 247-248, Jan. 2003.

[10] P. Regoliosi, A. Di Carlo, A. Reale, P. Lugli, M. Peroni, C. Lanzieri, and A. Cetronio, "Thermal resistance measurement of GaAs MESFETs by means of photocurrent spectrum analysis and comparison with simulations," Semicond. Sci. Technol., vol. 20, no. 2, pp. 135-139, Feb. 2005.

[11] P. Regoliosi, A. Reale, A. Di Carlo, P. Romanini, M. Peroni, C. Lanzieri, A. Angelici, M. Pirola, and G. Ghione, "Experimental validation of GaN HEMTs thermal management by using photocurrent measurements," IEEE Trans. Electron Devices, vol. 53, no. 2, pp. 182-188, Feb. 2006.

[12] G. Ghione and C. U. Naldi, "High-resolution self-consistent thermal modelling of multi-gate power GaAs MESFETs," in IEDM Tech. Dig., Dec. 3-6, 1989, pp. 147-150. Digital Object Identifier 10.1109/ IEDM.1989.74248.

[13] J. P. Landesman, E. Martin, and P. Braun, "Temperature distribution in power GaAs field effect transistors using spatially resolved photoluminescence mapping," in Proc. 7th Int. Symp. Phys. and Failure Anal. Integr. Circuits, Jul. 5-9, 1999, pp. 185-190.

\section{Concave-Suspended High- $Q$ Solenoid Inductors With an RFIC-Compatible Bulk-Micromachining Technology}

\author{
Lei Gu and Xinxin Li
}

\begin{abstract}
Presented in this brief is a concave-suspended solenoid inductor that can be post-CMOS integrated into a low-resistivity silicon substrate using a novel radio frequency integrated circuit (RFIC)-compatible micromachining technology. The three-mask processes include photoresist spray coating, $\mathrm{XeF}_{2}$ gaseous etching and copper electroplating, etc. The fabricated 11.5-turn inductor is measured with $2.96-\mathrm{nH}$ inductance at $5.35 \mathrm{GHz}$, where the peak $Q$-factor is as high as about 45 . Even with a compact layout $(50 \%$ area occupation saved), the 8.5 -turn inductor is still measured with $2.78 \mathrm{nH}$ and peak $Q$ of about 28 at $4.90 \mathrm{GHz}$. Both finite element simulation and shock testing results show that the suspended inductor is almost free of influence from environmental vibration and shock. Featuring high- $Q$ performance and robust properties, the RFIC-compatible inductors are promising for high-performance RFIC applications.
\end{abstract}

Index Terms-Bulk micromachining, $Q$-factor, radio frequency integrated circuit (RFIC), solenoid inductors.

\section{INTRODUCTION}

On-chip integrated high- $Q$ inductor is a key passive component in radio frequency integrated circuits (RFICs) such as voltage-controlled oscillators, low-noise amplifiers, filters, and impedance matching networks [1]. The microinductors can be categorized into planar spiral inductors [2]-[10] and solenoid inductors [11]-[16]. Many efforts have been focused on 2-D spiral inductors due to the good integration capability in standard silicon substrates. However, the aluminum planar spiral inductors generally exhibit a low $Q$-factor due to severe substrate loss and ohmic loss. In order to reduce the substrate loss and the metal resistance, efforts have been made on fabricating high- $Q$ spiral inductors, such as using porous silicon substrate [4], plastic substrate

Manuscript received July 12, 2006; revised December 13, 2006. This research is supported by Chinese 973 Project (2006CB300405). The review of this brief was arranged by Editor C.-Y. Lu.

The authors are with the State Key Laboratory of Transducer Technology, Shanghai Institute of Microsystem and Information Technology, Chinese Academy of Sciences, Shanghai 200050, China, and they are also with the Graduate University of Chinese Academy of Sciences, Beijing 100049, China (e-mail: xxli@mail.sim.ac.cn).

Digital Object Identifier 10.1109/TED.2007.892362 